\title{
Polarization of M2 Macrophages by Interaction between Prostate Cancer Cells Treated with Trichomonas vaginalis and Adipocytes
}

\author{
Hyo-Yeoung Chung ${ }^{1,2}$, Jung-Hyun Kim ${ }^{1,2}$, Ik-Hwan Han ${ }^{1,2}$, Jae-Sook Ryu ${ }^{1,2, *}$ \\ ${ }^{1}$ Department of Environmental Biology and Medical Parasitology, Hanyang University College of Medicine, Seoul 04763, Korea; \\ ${ }^{2}$ Department of Biomedical Science, Hanyang University Graduate School of Biomedical Science and Engineering, Seoul 04763, Korea
}

\begin{abstract}
Trichomonas vaginalis causes inflammation of the prostate and has been detected in tissues of prostate cancers $(\mathrm{PCa})$, prostatitis and benign prostatic hyperplasia. Obesity is a risk factor for PCa and causes a chronic subclinical inflammation. This chronic inflammation further exacerbates adipose tissue inflammation as results of migration and activation of macrophages. Macrophages are the most abundant immune cells in the PCa microenvironment. M2 macrophages, known as Tumor-Associated Macrophages, are involved in increasing cancer malignancy. In this study, conditioned medium (TCM) of PCa cells infected with live trichomonads contained chemokines that stimulated migration of the mouse preadipocytes (3T3-L1 cells). Conditioned medium of adipocytes incubated with TCM (ATCM) contained Th2 cytokines (IL-4, IL-13). Macrophage migration was stimulated by ATCM. In macrophages treated with ATCM, expression of M2 markers increased, while M1 markers decreased. Therefore, it is suggested that ATCM induces polarization of M0 to M2 macrophages. In addition, conditioned medium from the macrophages incubated with ATCM stimulates the proliferation and invasiveness of PCa. Our findings suggest that interaction between inflamed PCa treated with $T$. vaginalis and adipocytes causes M2 macrophage polarization, so contributing to the progression of PCa.
\end{abstract}

Key words:Trichomonas vaginalis, prostate cancer, adipocyte, macrophage, M2 polarization

\section{INTRODUCTION}

Trichomonas vaginalis is a protozoan parasite that causes trichomoniasis, a common sexually transmitted disease. The WHO reports that approximately 143 million people worldwide are infected with T. vaginalis, of which more than 52\% are male [1]. T. vaginalis has been detected in the urine of patients with chronic prostatitis and in tissues of patients with benign prostate hyperplasia and prostate cancer [2,3]. We showed previously that $T$. vaginalis infection caused an inflammatory response in prostate epithelial and stromal cells $[4,5]$, and that inflammatory mediators including IL-6 released from prostate epithelial cells infected with $T$. vaginalis increased the proliferation and invasiveness of prostate cancer cells [6]. However, it is not known whether T. vaginalis infection could

- Received 19 December 2019, revised 12 May 2020, accepted 12 May 2020.

*Corresponding author (jsryu@hanyang.ac.kr)

(c) 2020. Korean Society for Parasitology and Tropical Medicine

This is an Open Access article distributed under the terms of the Creative Commons Attribution Non-Commercial License (https://creativecommons.org/licenses/by-nc/4.0) which permits unrestricted non-commercial use, distribution, and reproduction in any

medium, provided the original work is properly cited. cause progression of prostate cancer.

Prostate cancer (PCa) is the most common cancer of men in the United States [7]. Various factors, such as age and diet, are known to affect the occurrence of PCa [8]. Obesity and overweight account for about $20 \%$ of cancers [9], and obese person have an increased risk of several types of cancer including prostate and breast cancer [9], and a poorer prognosis [10].

Adipose tissue consists of adipocytes, fibroblasts and macrophages, and is an important endocrine organ that secretes cytokines and adipokines [11]. The inflammatory response induced by obesity is known to increase cytokine production, and contributes to the development and aggravation of PCa in a paracrine manner [12]. In particular, CCL2 secreted by adipocytes induces macrophages to migrate into adipose tissues and causes chronic inflammation [13], leading to cancer malignancy [14,15].

Macrophages that migrate towards regions of inflammation differentiate into M1 or M2 macrophages depending on the microenvironment, and the 2 types of macrophage have different roles. Within tumor microenvironments macrophages primarily exhibit M2-type functional profiles, and this pre- 
ferred polarization is due to stimulation by Th2 cytokines [16]. Adipocytes produce IL-4 and IL-13 cytokines, which promote M2 polarization of macrophages around adipocytes. M2 macrophages affect growth, invasion, and metastasis of cancer cells [17]. They are also involved in the progression of PCa and angiogenesis. Although they aggravate prostate cancer [18], there is no known effect of M2 macrophages on the malignancy of prostate cancer cells infected with T. vaginalis. In this experiment, we observed the immunologic function of adipocyte and macrophage in the prostate cancer cells treated with $T$. vaginalis. Therefore, in this study, we investigated whether adipocytes reacted with the culture supernatant of prostate cancer cells infected with T. vaginalis induced M2 polarization of M0 macrophages and whether this resulted in increased proliferation and invasiveness of the cancer cells. Our findings suggest that inflamed prostate cancer cells infected with T. vaginalis induce adipocyte migration and activation, and the stimulated adipocytes produce adipokines such as IL-4, IL-13, CCL2, and IL-6, leading to proliferation and increased invasiveness of the prostate cancer cells as a result of the M2 macrophage polarization.

\section{MATERIALS AND METHODS}

\section{Parasite and cell lines}

Trichomonas vaginalis isolate T016 was grown in trypticaseyeast extract-maltose medium (TYM) supplemented with 10\% heat-inactivated horse serum (Life Technologies, Carlsbad, California, USA).

The mouse prostate cancer cells (TRAMP-C2) were a gift from Prof. Jung Han Yoon Park (Hallym University, Chuncheon, Korea) and cultured in Dulbecco's Modified Eagle's Medium (DMEM;GE Healthcare Life Sciences, Logan, Utah, USA) containing penicillin and streptomycin (WelGENE, Gyeongsangbukdo, Korea) and supplemented with 10\% fetal bovine serum (FBS; GE Healthcare Life Sciences, Utah, USA) in a $5 \% \mathrm{CO}_{2}$ humidified incubator at $37^{\circ} \mathrm{C}$.

The mouse preadipocyte line cells (3T3-L1) were a gift from Prof. Jae-woo Kim (Yonsei University, Seoul, Korea) and was grown in DMEM containing 10\% bovine calf serum (BCS; Thermo Fisher Scientific, Massachusetts, USA) and penicillinstreptomycin solution at $37^{\circ} \mathrm{C}$ in a $5 \% \mathrm{CO}_{2}$ incubator. Preadipocytes were differentiated into adipocytes for the study. To induce differentiation, 3T3-L1 preadipocytes $\left(4 \times 10^{4}\right.$ cells/well $)$ were seeded in 24-well plates (Corning, New York, USA).
When they had grown to confluence, the DMEM medium containing $10 \%$ BCS was removed and changed to adipocyte differentiation medium containing 10\% FBS, $0.5 \mathrm{mM}$ 3-isobutyl-1-methylxanthine, $1 \mu \mathrm{M}$ dexamethasone and $10 \mu \mathrm{g} / \mathrm{ml}$ insulin for 3 days. The cells were then cultured in DMEM containing 10\% FBS and insulin for another 2 days, followed by adipocyte maintenance DMEM containing 10\% FBS supplemented with penicillin-streptomycin solution, following a standard protocol.

To identify lipid droplets accumulated in the cytoplasm of mature adipocytes, Oil-Red $\mathrm{O}$ staining was performed as described [19]. The 3T3-L1 cells were incubated and differentiated in sterile cover glasses (Paul Marienfeld GmbH \& Co., KG, Lauda-Konigshofen, Germany). Mature adipocytes were fixed with $10 \%$ formalin for $10 \mathrm{~min}$ and dried completely. The fixed cells were stained with Oil-Red O in 60\% isopropyl alcohol solution for $1 \mathrm{hr}$ at room temperature, and the presence of lipid droplets was visualized under a light microscopy, and photomicrographs obtained.

The mouse macrophage cell line (RAW264.7) cells were purchased from the Korean Cell Line Bank (KCLB, Seoul, Korea) and cultured in DMEM containing penicillin and streptomycin plus $10 \%$ FBS. All mouse cell lines were maintained in a $5 \% \mathrm{CO}_{2}$ humidified incubator at $37^{\circ} \mathrm{C}$.

\section{Preparation of conditioned media and treatments}

For conditioned medium preparation, TRAMP-C2 prostate cancer cells $\left(5 \times 10^{4}\right.$ cells/well $)$ were seeded in 12 -well plates (Corning) for $24 \mathrm{hr}$. The medium was changed to serum-free medium, and the cells were incubated with or without live $T$. vaginalis in a ratio of 1:10 for $3 \mathrm{hr}$. Conditioned medium was collected, filtered through a $0.2 \mu \mathrm{m}$ filters (GVS Filter Technology, Indianapolis, USA) to remove cell debris, and stored at $-20^{\circ} \mathrm{C}$. Culture supernatants incubated with T. vaginalis were named as trichomonad-conditioned medium (TCM) and without T. vaginalis as conditioned medium (CM).

Adipocyte-conditioned media (ATCM and ACM) were collected from fully differentiated adipocytes, cultured with and without TCM, respectively, for $6 \mathrm{hr}$. The supernatants were collected, filtered through a $0.2 \mu \mathrm{m}$ filter (GVS) and stored at $-20^{\circ} \mathrm{C}$.

The RAW264.7 cells $\left(5 \times 10^{4}\right.$ cells/well) were seeded in 12well plates (Corning) in DMEM medium for stabilization. After $24 \mathrm{hr}$, they were incubated with 10\% ATCM or 10\% ACM for $24 \mathrm{hr}$, and supernatants were collected and stored in the 
same way, yielding conditioned media M-ACM and M-ATCM, respectively. For M2-type macrophage polarization, RAW264.7 cells were treated with $20 \mathrm{ng} / \mathrm{ml} \mathrm{IL-4}$ and $20 \mathrm{ng} / \mathrm{ml} \mathrm{IL-13}$ (PROSPEC, Ness-Ziona, Israel) for $24 \mathrm{hr}$. In contrast, to generate M1-type macrophages, RAW264.7 cells were treated with $20 \mathrm{ng} / \mathrm{ml}$ IFN- $\gamma$ (PROSPEC) and $100 \mathrm{ng} / \mathrm{ml}$ lipopolysaccharide (LPS;Sigma-Aldrich, St. Louis, USA) for $24 \mathrm{hr}$. Final concentrations of the conditioned media used for proliferation and invasion assays were adjusted to $10 \%$ with cell culture medium. All cells were harvested for further analysis.

\section{Measurement of cytokine levels}

CXCL1 and CCL2 chemokine levels produced by TRAMPC2 cells stimulated with live T. vaginalis were measured in cell culture medium using the mouse CXCL1 ELISA DuoSet (R\&D System, Minneapolis, Minnesota, USA) and CCL2 ELISA set (BD, Bioscience Pharmingen, San Diego, California, USA). Concentrations of other adipokines were measured with a mouse IL-4 ELISA kit (Enzo Life Science, Farmingdale, New York, USA), mouse CCL2 ELISA set (BD) and mouse IL6 ELISA DuoSet (R\&D System). ELISA DuoSets (R\&D System) for mouse IL-10, IL1 $\beta$, and IL6 were used to detect the respective cytokines in RAW264.7 cell supernatants.

\section{Cell migration assay}

Migratory abilities of 3T3-L1 preadipocytes and RAW264.7 cells were determined using 24-well plates (Corning) with 8 and $5 \mu \mathrm{m}$ polycarbonate pore membrane inserts (Corning), respectively. For preadipocytes migration, the lower wells were filled with $350 \mu \mathrm{l}$ control (serum-free medium) or conditioned media (TCM or CM). For RAW264.7 cell migration, the lower wells were filled with $350 \mu \mathrm{l}$ control (serum-free medium) or conditioned media (ATCM, ACM, TCM or CM). Polycarbonate pore membrane inserts were placed over the lower wells. To promote adhesion of the cells, the filters were pretreated with human plasma fibronectin $(100 \mu \mathrm{g} / \mu \mathrm{l}$, SigmaAldrich) overnight at $4^{\circ} \mathrm{C}$ and air-dried for $30 \mathrm{~min}$. The upper wells were filled with $200 \mu \mathrm{l}$ of cells $\left(2 \times 10^{5}\right.$ cells/well $)$ in serum-free medium. The plates were incubated for $24 \mathrm{hr}$ at $37^{\circ} \mathrm{C}$. After incubation, cells that did not migrate through the pores were removed with a cotton swab. Cells that had migrated to the underside of the membrane were fixed in methanol for 10 $\mathrm{min}$, and stained with Giemsa stain solution for $20 \mathrm{~min}$. Five randomly-selected fields per membrane were counted under a light microscope, and the chemotactic index was calculated from the number of cells that migrated relative to the control. The calculation formula is as follows; No. of migrated cells in experimental group/No. of migrated cells in control group.

\section{Western blot analysis}

Binding of IL-4 or IL-13 to the IL-4 receptor of macrophages results in M2 polarization of the macrophages [18]. Therefore, we tested for expression of IL-4Ra on the macrophages. To confirm IL-4Ra expression, RAW264.7 cells were seeded at $1 \times 10^{5}$ cells/well in 12-well plates. After overnight incubation, the cells cultured in 10\% conditioned media (ATCM, ACM, TCM or CM) for $3 \mathrm{hr}$, harvested and lysed in PRO-PREP protein extraction solution (iNtRON Biotechnology, Seongnam, Korea), and protein concentrations were measured with the Bradford assay. The membranes were blocked with $3 \%$ bovine serum albumin and probed with anti-IL-4Ra polyclonal antibody (1:2,000; Abbkine, Wuhan, China) overnight at $4^{\circ} \mathrm{C}$, followed by goat anti-rabbit IgG polyclonal antibody $(1: 10,000$; ADISAB-300-J, Enzo Life Sciences) for $1 \mathrm{hr}$ at room temperature. The blots were visualized with Chemiluminescent Sensitive Plus HRP Microwell and/or Membrane Substrate (SurModics, Eden Prairie, Minnesota, USA), and signals were measured with a Chemi-Doc (Bio-Rad, California, California, USA).

\section{Reverse transcription PCR (RT-PCR) and quantitative real-time PCR (Q-PCR)}

Levels of mRNA in TRAMP-C2 cells $\left(5 \times 10^{4}\right.$ cells/well $)$ and 3T3-L1 cells $\left(4 \times 10^{4}\right.$ cells/well $)$ were measured by RT-PCR. Levels of CXCL1 and CCL2 mRNAs in TRAMP-C2 cells were measured after incubation with live T. vaginalis for $3 \mathrm{hr}$, and IL4, IL-13, CCL2, and IL-6 mRNAs in mature adipocytes were measured after incubation in 100\% conditioned media (CM or TCM) for $6 \mathrm{hr}$. M1 and M2 marker mRNAs were measured in macrophage after incubation in 10\% conditioned media (ATCM, ACM, TCM or CM) for $1 \mathrm{hr}$. Total RNA was extracted from cells using Trizol reagent (Favorgen Biotech Corp, Kaohsiung, Taiwan) and the CDNA was used as template for subsequent PCR amplification using following gene-specific primers. CXCL1; 5'-CAC CCA AAC CGA AGT CAT AG-3', 5'-AAG CCA GCG TTC ACC AGA-3', CCL2; 5'-GCT ACA AGA GGA TCA CCA GC-3', 5'-TGT CTG GAC CCA TTC CTT CT-3', IL-4; 5' -AGA ATG AAA GGC CCC AAA GT-3', 5'-CAT GGC TTG GGT ACA GGT CT-3', IL-6; 5'-CCA CTT CAC AAG TCG GAG GCT TA-3', 5'-CCA CTT CAC AAG TCG GAG GCT TA-3', IL-13; 5' -TGG CTG TTG GCT TCT TTT CT-3', 5'-TGG CTG TTG GCT 
TCT TTT CT-3', Arginase1; 5'-AAG CTG GTC TGC TGG AAA AA-3', 5' '-TCT TCA GCG TGG ATC TGT TG-3', CD206; 5'-AGG TCT TGT GGG TCA CTT GG-3', 5'-GGA TCT GGA AGG GAC TCT CC-3', IL-10; 5'-TCA GAG CTC CTG GAA CTG GT-3', 5' -CAC CTG TGT CAA CCC TTC CT- $3^{\prime}$, TNF- $\alpha$; $5^{\prime}$-TGG GAC AGT GAC CTG GAC TGT-3' ${ }^{\prime} 5^{\prime}$-TTC GGA AAG CCC ATT TGA GT-3', iNOS; 5'-CCC CAC AGA GTG GTG TTC TT-3', 5'-CCC TGG CTA GTG CTT CAG AC-3', CXCL10; 5'-TCC TTG TCC TCC CTA GCT CA-3', 5'-ATA ACC CCT TGG GAA GAT GG-3', GAPDH; 5'-AAT ACG GCT ACA GCA ACA GG-3', 5' -TTG GGA TAG GGC CTC TCT TG-3'.

For quantitative real-time PCR in macrophages, target mRNAs were amplified using LightCycler 480 SYBR Green I Master (Roche Life Science, Mannheim, Germany) and analyzed with LightCycler 480 Software (Roche Life Science). PCR conditions were: 45 cycles at $95^{\circ} \mathrm{C}$ for $5 \mathrm{~min}, 95^{\circ} \mathrm{C}$ for $10 \mathrm{sec}$, $60^{\circ} \mathrm{C}$ for $10 \mathrm{sec}$ and $72^{\circ} \mathrm{C}$ for $10 \mathrm{sec}$.

\section{Proliferation assay}

To examine the effect of conditioned media on the proliferation of prostate cancer cells, TRAMP-C2 cells $\left(3 \times 10^{3} \mathrm{cells} /\right.$ well $)$ were seeded in 96-well plates and cultured in DMEM with 10\% FBS. After $24 \mathrm{hr}$, they were transferred to 10\% conditioned media (M-ATCM, M-ACM, ATCM or TCM) or control medium (DMEM) and incubated for $24 \mathrm{hr}$. After incubation, cell growth was analyzed with the CCK-8 assay: CCK-8 reagent (Enzo Life Sciences) was added to each well for an additional $2 \mathrm{hr}$ before obtaining spectrophotometric readings. The CCK- 8 reagent is reduced by dehydrogenases to yield a water-soluble formazan dye, and the amount of the dye generated is directly proportional to the number of living cells. When the O.D. value by the control cells was set to 100 , the degree of proliferation of the experimental group was relatively calculated.

\section{Invasiveness assay}

Invasion of prostate cancer cells (TRAMP-C2) was investigated using 24-well plates (Corning) with $8 \mu \mathrm{m}$ pore polycarbonate membrane inserts (Corning pre-coated with Matrigel (200-300 $\mu \mathrm{g} / \mathrm{ml}$, SigmaAldrich) for $2 \mathrm{hr}$ at $37^{\circ} \mathrm{C}$; the matrigel matrix layer was not allowed to dry out before use. Conditioned media ( $350 \mu \mathrm{l} ; \mathrm{M}$-ATCM, M-ACM, ATCM or TCM) were used as chemoattractants in the lower chamber while TRAMP$\mathrm{C} 2$ cells $\left(2 \times 10^{5}\right.$ cells/well) were seeded in the upper chamber. The plates were incubated overnight at $37^{\circ} \mathrm{C}$ for $24 \mathrm{hr}$. After that, the invaded cells were fixed in methanol for $10 \mathrm{~min}$ and stained with Giemsa stain solution for $20 \mathrm{~min}$. Five randomly selected fields per membrane were counted under a light microscope. Invasiveness was calculated from the number of cells that had migrated relative to the control group with DMEM. Invasiveness was expressed as a ratio: number of the invaded cells in the experimental group divided with number of cells invaded in the control group.

\section{Statistical analyses}

Statistical analyses were performed using SPSS Statistical Software, version 24 (IBM, Chicago, Illinois, USA). The MannWhitney $\mathrm{U}$ test was used for comparisons, and 2-tailed $P$-values $<0.05$ were considered statistically significant. Values are presented as means \pm SEMs of 3-4 independent experiments.

\section{RESULTS}

\section{Chemokine production by TRAMP-C2 cells incubated with live $T$. vaginalis}

We first examined chemokines produced by TRAMP-C2 cells treated with T. vaginalis (1:10) for $3 \mathrm{hr}$. The TRAMP-C2 cells released CXCL1 (95.9 pg/ml) and CCL2 (195.9 pg/ml), and chemokine production was significantly increased in comparison with cells incubated without T. vaginalis $(P<0.05$; Fig. 1A). When TRAMP-C2 cells were stimulated with live $T$. vaginalis $(1: 10)$ for $30 \mathrm{~min}$, levels of the chemokine mRNAs were also significantly upregulated in comparison to the control. (Fig. 1B). The culture supernatants of the TRAMP-C2 cells incubated with and without $T$. vaginalis were named TCM ( $T$. vaginalis-conditioned medium) and CM (conditioned medium), respectively.

\section{Preadipocyte migration by conditioned medium from trichomonad-stimulated TRAMP-C2 cells}

To determine whether conditioned medium from trichomonad-stimulated TRAMP-C2 cells could induce migration of preadipocytes, 3T3-L1 cells were incubated with CM or TCM for $24 \mathrm{hr}$. The migration of cells was assessed by counting the number of cells that migrated through a membrane to the lower well after contact with conditioned medium. Chemotaxis of 3T3-L1 cells induced by TCM was significantly greater than that induced by CM or DMEM. These results suggest that TCM from inflamed TRAMP-C2 cells infected with T. vaginalis containing CXCL1 and CCL2 has a role in the recruitment of 3T3-L1 cells $(P<0.05$; Fig. 1C). 
A
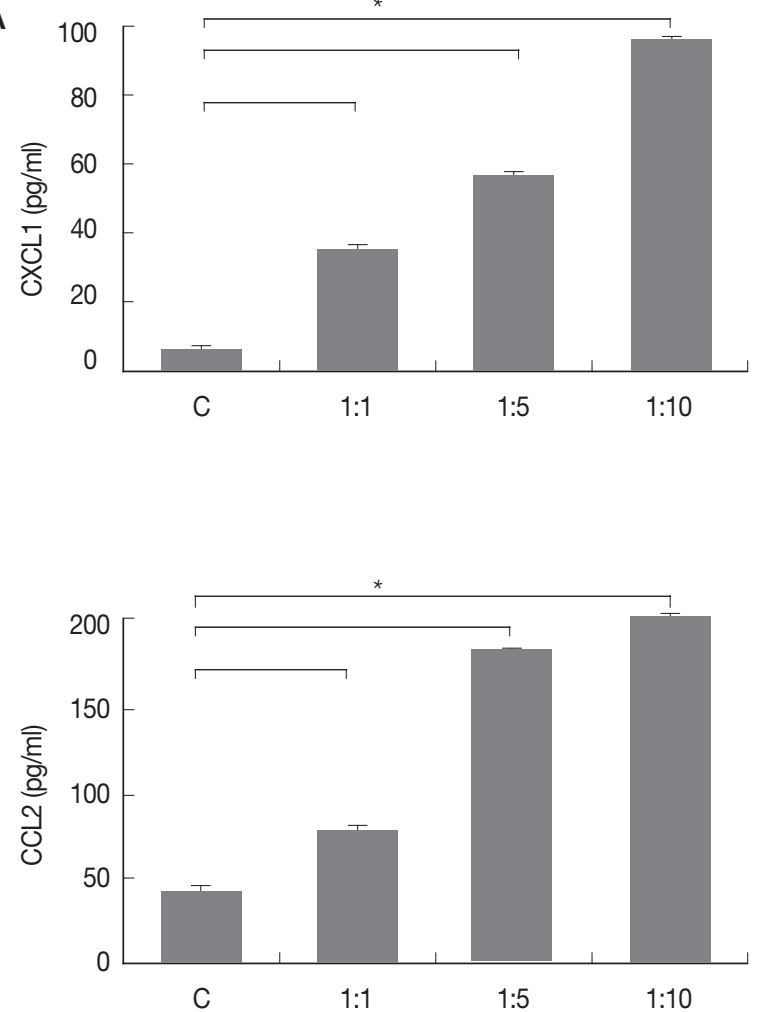

B
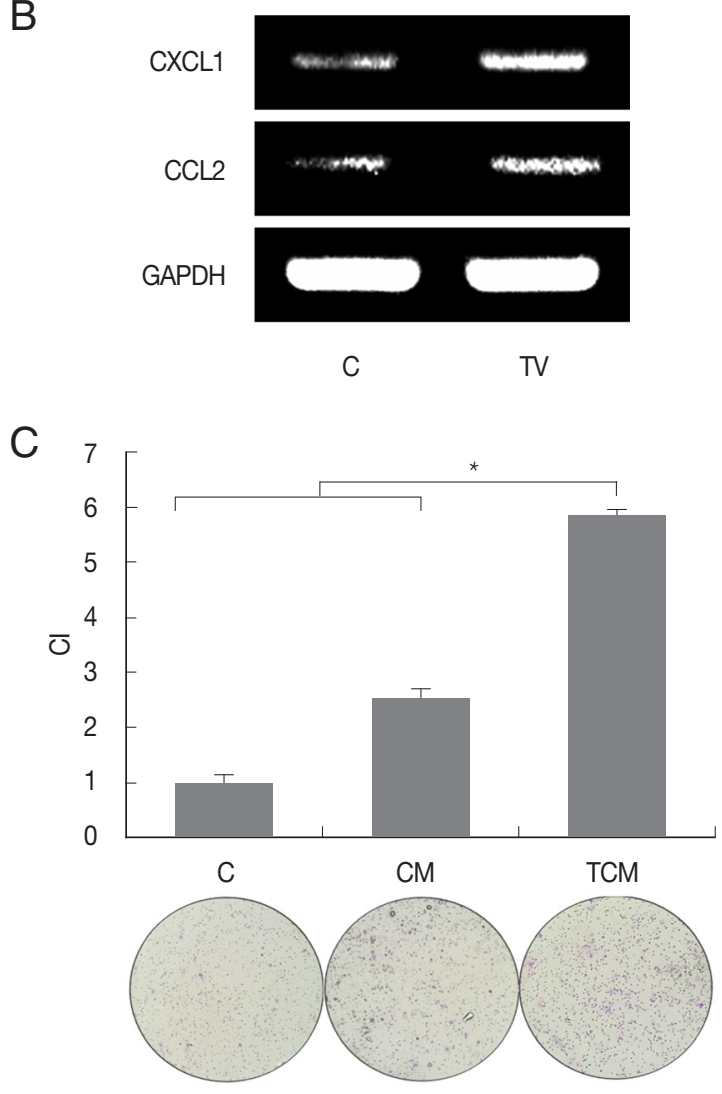

Fig. 1. Migration of preadipocytes (3T3-L1) into the inflammatory response (TCM) of prostate cancer cells infected with Trichomonas vaginalis. To examine chemokine production by prostate cancer cells (TRAMP-C2 cell line), TRAMP-C2 cells were incubated with increasing numbers of live T. vaginalis (Tv). (A) Production of cytokine CXCL1 and CCL2 was measured by ELISA and (B) their mRNA was confirmed by RT-PCR. (C) To examine preadipocytes migration induced by TCM, chemotaxis assay was done. This graph shows the relative movement by CM and TCM when the migration by DMEM is 1. C: control (DMEM). CM: culture supernatant of prostate cancer cells. TCM: culture supernatant of prostate cancer cells co-cultured with $T$. vaginalis. Cl: chemotactic index. ${ }^{*} P<0.05$ vs I:10 (TRAMPC2: T. vaginalis). ${ }^{\star} P<0.05(\mathrm{C})$.

\section{Adipokine production by adipocytes stimulated with TCM}

We examined whether adipocytes could manifest an inflammatory response to TCM. 3T3-L1 preadipocytes were matured in adipocyte differentiation medium, and the formation of mature adipocytes was confirmed by lipid staining using OilRed O (Fig. 2A). When the adipocytes were stimulated with TCM, they released adipokines including IL-4, CCL2 and IL-6, and IL-4, IL-13, CCL2, and IL-6 mRNAs increased $(P<0.05$; Fig. 2B, C). Since IL-4, IL-13, and IL-6 are well- known M2 macrophage inducers, this result confirmed that adipokine production, especially of M2 macrophage inducers, increased in inflamed adipocytes induced by TCM. Mature adipocytes incubated with $\mathrm{CM}$ or TCM yielded conditioned media ACM and ATCM, respectively.

\section{Migration of macrophages toward ATCM}

As shown in (Fig. 2D), ATCM induced migration of RAW264.7 macrophages more effectively than ACM, TCM, CM, and DMEM. These results suggest that ATCM, resulting from crosstalk between adipocytes and TRAMP-C2 cells treated with $T$. vaginalis, stimulates the migration of macrophages more strongly than TCM $(P<0.05 ;$ Fig. 2D).

\section{Polarization of M2 macrophages induced by ATCM}

IL-4 or IL-13 have been reported to be involved in the polarization of M2 macrophages [18]. Since the results in (Fig. 2) showed that IL-4 and IL-13 were present in ATCM, we asked whether ATCM caused polarization of macrophages. We treated RAW264.7 cells with 10\% CM, TCM, ACM or ATCM, and as controls for M2 and M1 macrophage polarization, we used 
A

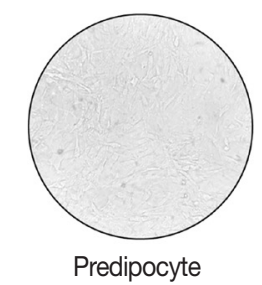

(3T3-L1 cell)

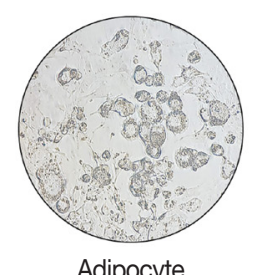

Adipocyte

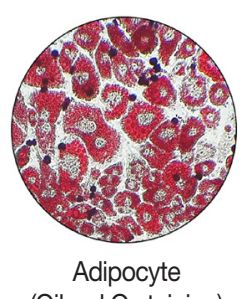

(Oil red O staining)
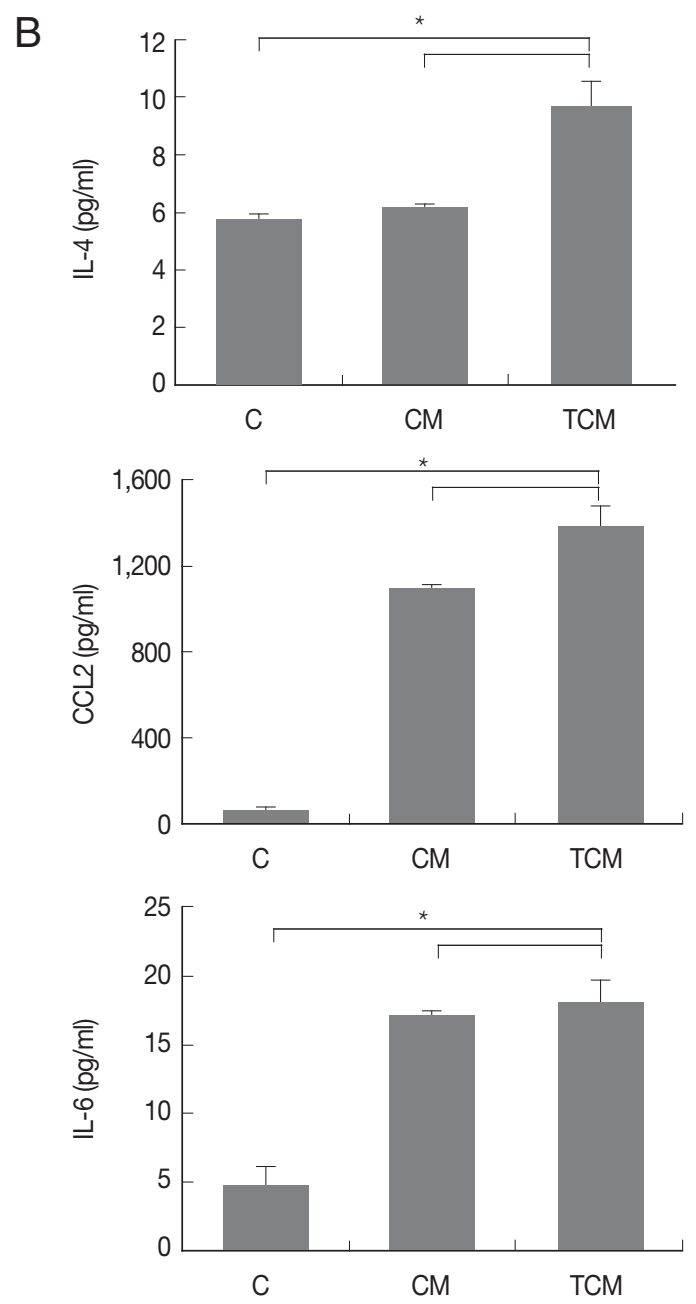

C
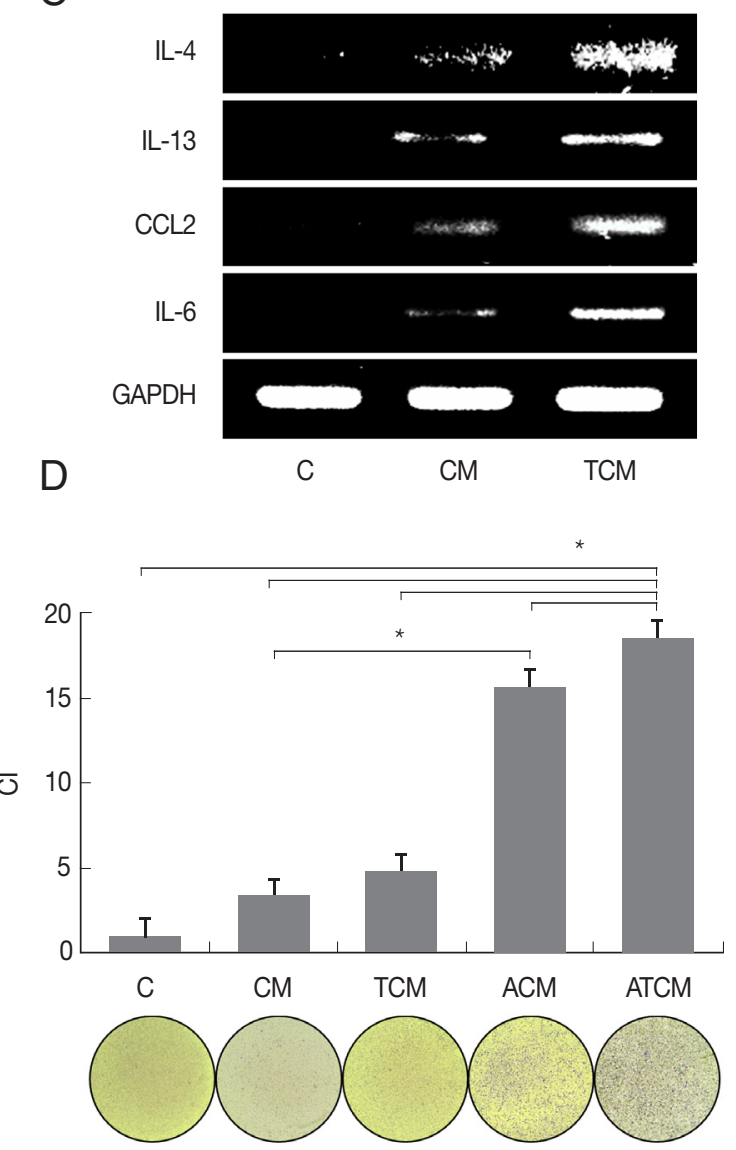

Fig. 2. Adipokines secreted by adipocyte stimulated with TCM increased the migration of macrophage (RAW264.7). Spindle shape of preadipocyte (left), and mature adipocytes (center) were stained with Oil-Red O solution (right). (A) Lipid accumulation of the adipocytes was observed in red. (B) Expression of cytokines and (C) mRNA by adipocytes stimulated with TCM was measured by ELISA and RT-PCR, respectively. To investigate RAW264.7 macrophages migration caused by ATCM, chemotaxis assay was done. (D) The graph shows relative migration of macrophage in compared with DMEM. C: control (DMEM). CM: culture supernatant from prostate cancer cells. TCM: culture supernatant from prostate cancer cells stimulated with $T$. vaginalis. ACM: culture supernatant from adipocytes stimulated with $\mathrm{CM}$. ATCM: culture supernatant from adipocytes stimulated with TCM. Cl: chemotactic index. ${ }^{\star} P<0.05$.
IL-4 and IL-13 for M2 macrophages, and IFN- $\gamma$ and LPS for M1 macrophages (Fig. 3A). In RAW264.7 cells exposed to ATCM, levels of the M2 macrophage markers arginase1, CD206, CXCL1, and IL-10 were higher than in cells exposed to ACM, TCM, CM or medium. In contrast, ATCM induced lower levels of M1 markers including TNF- $\alpha$, CXCL10, iNOS mRNAs, and IL- $1 \beta$ and IL-6 $(P<0.05$; Fig. 3B). This suggested that ATCM, which contains IL-4 and IL-13, induces M2 polarization of macrophages. Next, we investigated whether IL-4Ra, the receptor for IL-4 and IL-13, is involved in the M2 polarization induced by ATCM. When RAW264.7 cells were incubated in 10\% ATCM for $3 \mathrm{hr}$, expression of IL-4Ra was higher than incubation with CM, TCM, or ACM, and its level was similar to that obtained in the positive control with recombinant IL-4 and IL-13 (Fig. 3C). Therefore, it is likely that the IL-4 or IL-13 contained in ATCM induces M2 polarization by binding to IL4Ra on the macrophages. RAW264.7 macrophages incubated with ACM and ATCM yielded conditioned media M-ACM and 
A
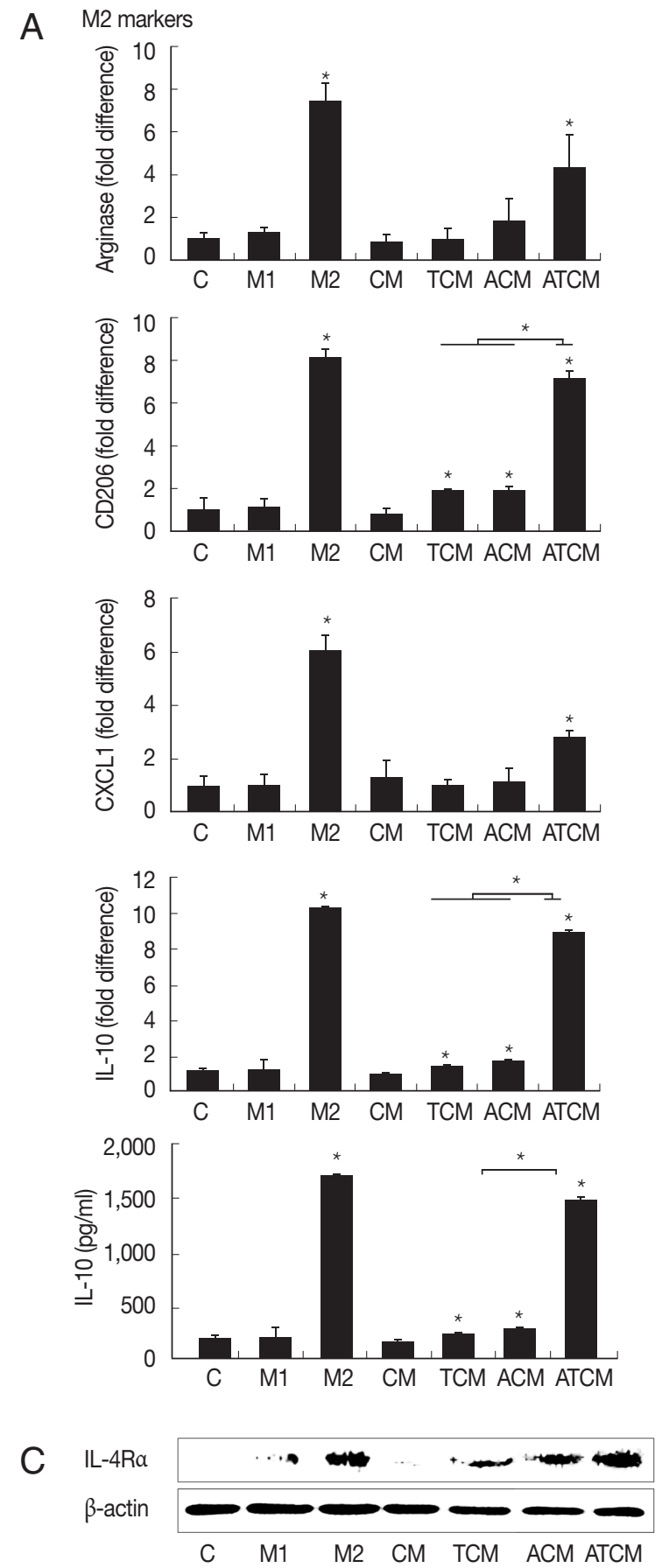
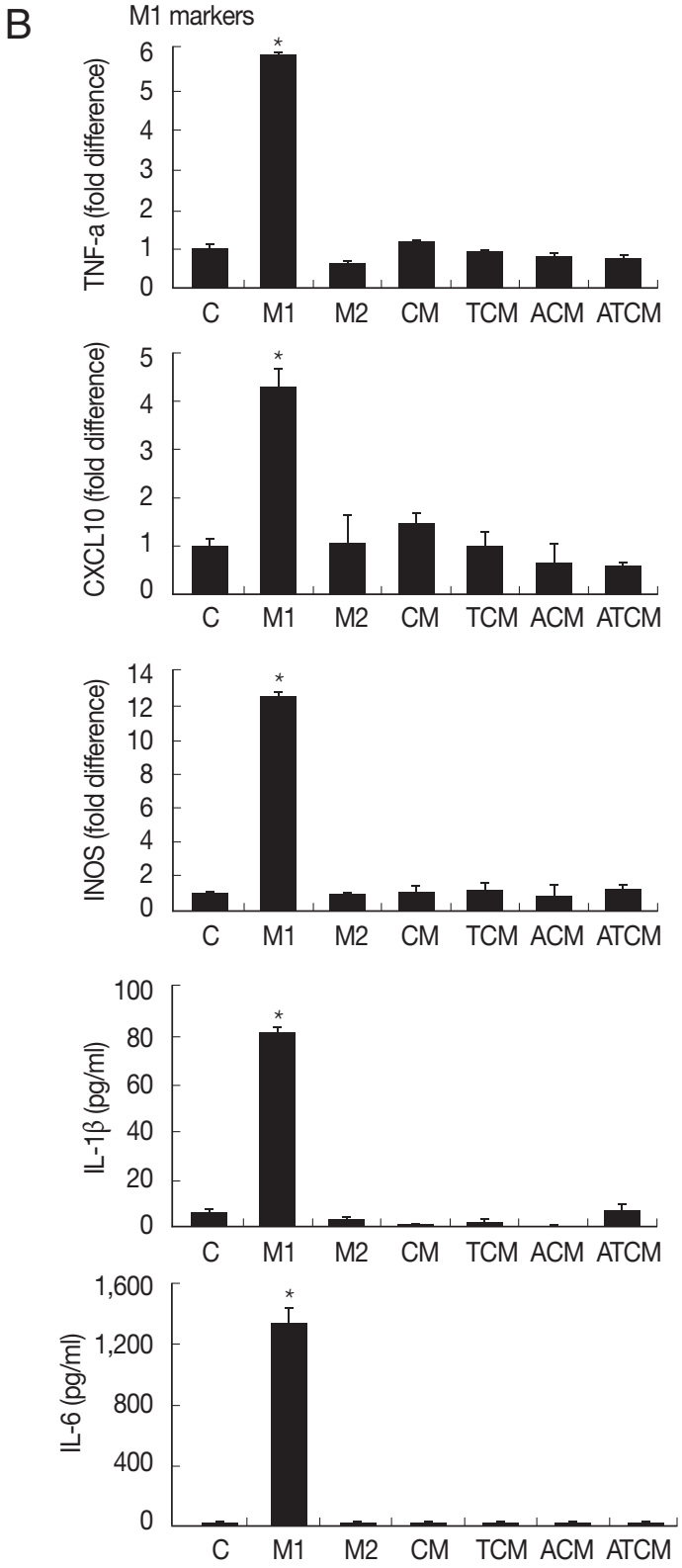

Fig. 3. ATCM polarized M0 macrophages to M2 macrophages and induced the expression of IL-4Ra in macrophages. In order to investigate whether ATCM induces polarization of M0 macrophage into M2 macrophages, RAW264.7 cells were stimulated with 10\% ATCM for $1 \mathrm{hr}$ (A\&B; mRNA), $24 \mathrm{hr}$ (A\&B; protein) or $3 \mathrm{hr}(\mathrm{C})$. The expression of M2-associated genes (Arginase1, CD206, CXCL1 and IL-10) and the M1-associated gene (TNF-a, CXCL10 and iNOS) were measured by real-time PCR. Production of M2-associated cytokine IL10 , and the M1-associated cytokines, including IL-1 $\beta$ and IL-6 was measured by ELISA. (A) When macrophages were treated with M2 activator, an increase in the expression of the M2 macrophage phenotype was observed. (B) M1 activator- $\neg$ treated macrophages were observed to have increased M1 marker expression. (C) IL-4Ra protein level in macrophages treated with $10 \%$ ATCM was confirmed by Western blotting. Equal protein loading was confirm using $\beta$-actin. C: control (DMEM). CM: culture supernatant from prostate cancer cells. TCM: culture supernatant from prostate cancer cells stimulated with T. vaginalis. ACM: culture supernatant from adipocytes stimulated with CM. ATCM: culture supernatant from adipocytes stimulated with TCM. ${ }^{*} P<0.05$. 

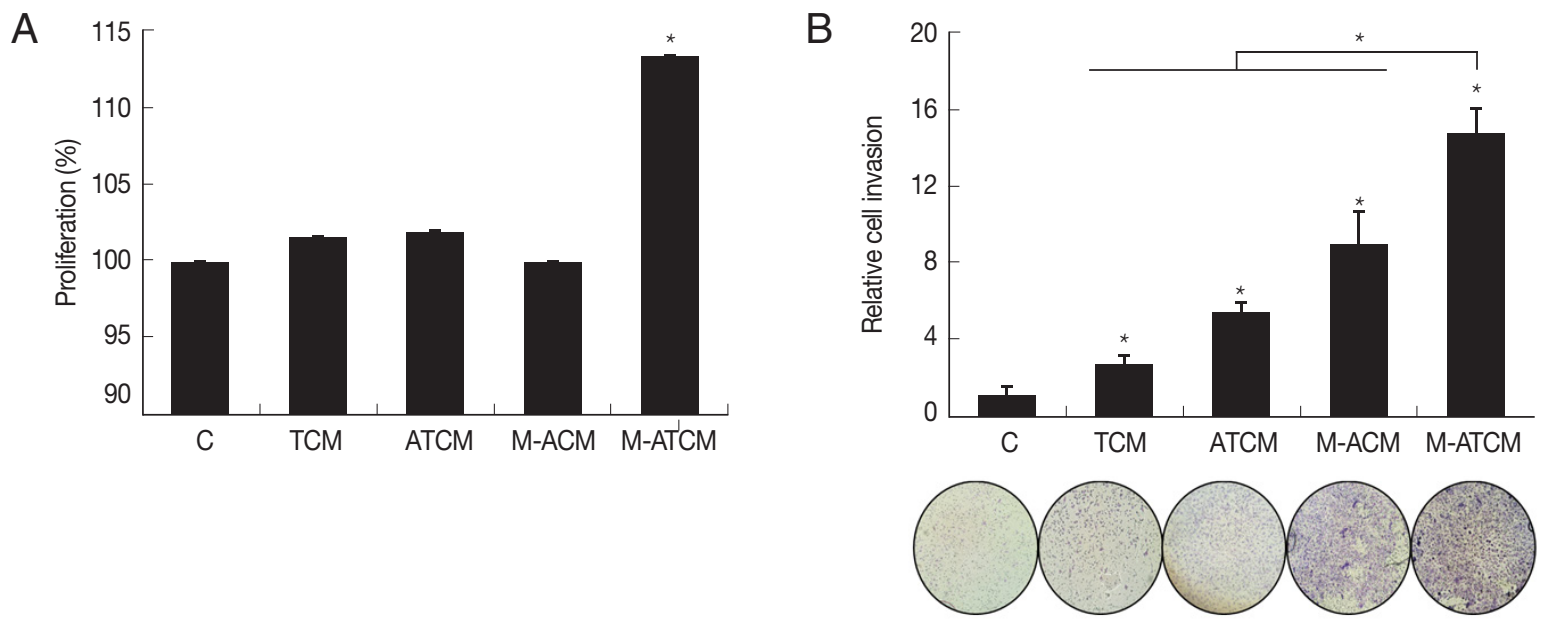

Fig. 4. M-ATCM increased proliferation and invasiveness of prostate cancer cells (TRAMP-C2). (A) To investigate proliferation and invasiveness induced by M-ATCM, prostate cancer cells (TRAMP-C2) were incubated with conditioned media (TCM, ATCM, M-ACM or MATCM) for 24 hr. (B) TRAMP-C2 cells were invaded to M-ATCM more than the other conditioned media (TCM, ATCM or M-ACM). C: control (DMEM). CM: culture supernatant from prostate cancer cells. TCM: culture supernatant from prostate cancer cells stimulated with T. vaginalis. ACM: culture supernatant from adipocytes stimulated with CM. ATCM: culture supernatant from adipocytes stimulated with TCM. M-ACM: culture supernatant of RAW264.7 cells stimulated with ACM. M-ATCM: culture supernatant of RAW264.7 stimulated cells with ATCM. * $P<0.05$.

\section{M-ATCM, respectively.}

\section{Proliferation and invasiveness of prostate cancer cells induced by M-ATCM}

We investigated whether M-ATCM stimulated the proliferation of prostate cancer cells using the CCK- 8 assay, and found that after $24 \mathrm{hr}$ of stimulation, TRAMP-C2 cell numbers were higher with M-ATCM than with M-ACM, ATCM or TCM. Thus, M-ATCM does stimulate the proliferation of prostate cancer cells $(P<0.05 ;$ Fig. 4A).

To test whether M-ATCM also affected prostate cancer cell invasiveness, we conducted a Matrigel invasion assay using TRAMP-C2 cells (see Methods). As shown in (Fig. 4B), MATCM stimulated the invasiveness of TRAMP-C2 cells more than DMEM, TCM, ATCM, or M-ACM $(P<0.05)$. Therefore, $M$-ATCM could cause progression of prostate cancers by inducing the proliferation and invasiveness of prostate cancer cells.

\section{DISCUSSION}

T. vaginalis is generally known as a surface-dwelling parasite. However, it has been detected in endo-epithelial cells and subepithelial tissue, which suggests that it is invasive. In our previous study, we confirmed by intraurethral injection [20], that $T$. vaginalis causes prostatitis in the rat, and proliferation of pros- tate epithelial and stromal cells. The proliferation of prostate stromal cells induced by inflammatory prostate epithelial cells infected with T. vaginalis occurred as a result of interaction with mast cells [21]. In turn, IL-6 released by proliferating stromal cells caused the proliferation of prostate epithelial cells by stromal-epithelial interaction [22]. In this study, PCa cells infected with $T$. vaginalis showed inflammatory reaction by production of CXCL1 and CCL2 (Fig. 1).

Adipose tissue is the largest endocrine organ, and it affects systemic metabolism by secreting adipokines. Adipocytes are the most vital cells in adipose tissue. When prostate cancer cells were cultured in the presence of adipocytes, their growth rate increased, and cytokines such as VEGF were produced [23]. Therefore, factors derived from adipocytes are thought to have an effect on the progression of prostate cancer. Finley et al. suggested that the production of inflammatory cytokines by the adipose tissues around the prostate increases and that these inflammatory mediators aggravate the prostate cancer [24]. Also Xie et al. found that the pre-adipocytes could be recruited more easily to PCa than its surrounding normal prostate tissue [25]. The result was line with the present study that migration of pre-adipocyte was increased by $\mathrm{CM}$ and TCM than the control medium. Interestingly, TCM from T. vaginalis infected PCa caused significantly increased migration of adipocyte than $\mathrm{CM}$. This means inflammatory response by T. vag- 
inalis infection may play a role on chemotaxis of adipocytes (Fig. 1C).

Recent studies suggest that obesity may represent a state of chronic, low-grade inflammation [10]. This chronic inflammation is known to increase the inflammation of adipose tissues through infiltration and accumulation of inflammatory cells. Hence inflammation may be expected to contribute to the development and progression of certain cancers [26]. Macrophages are the most abundant immune cells in the microenvironment of prostate cancers [27]. The number of macrophages in the adipose tissues of the obese mouse model increased, and the movement of macrophages toward adipocytes increased with the degree of obesity [28].

In this study, we asked if inflammatory mediators from prostate cancer cells infected with T. vaginalis in obese conditions could cause migration of macrophages. We found that when adipocytes were activated in response to TCM (culture supernatant of prostate cancer cells infected with live T. vaginalis), macrophage migration was more extensive than in response to CM or TCM alone. This shows that adipocytes may be involved in the migration of macrophages, in line with the evidence that macrophages migrate and infiltrate into inflamed adipose tissue [29]. In addition, the fact that ATCM stimulated migration of macrophages more than ACM (Fig. 2) implies that the inflammatory response to T. vaginalis infection increased macrophage migration.

Macrophages are recruited into tumors by inflammatory cytokines and chemokines. CCL2 is one of the key chemokines involved in attracting monocytes and macrophages, and it has been found to circulate at higher levels in obesity. Moreover, adipose cells are major contributors to CCL2 levels [30,31], and expression of CCL2 along with numbers of macrophages increases in the prostate cancer tissue of obese mice [32]. In this study, CCL2 produced from adipocyte activated by TCM attracted macrophages more strongly than ACM, which contained less CCL2 (Fig. 2C). This confirms that adipocytes producing CCL2 can induce macrophage migration.

Macrophages can be polarized in response to signals in certain microenvironments and show distinct heterogeneity of function [33]. Polarization is generally classified as M1 and M2 type. M1, or classically activated macrophages, are induced by the proinflammatory mediators (LPS and IFN-y). M1-type macrophages have anti-tumor properties and produce high levels of TNF- $\alpha$, IL-1 $\beta$, and IL- 6 . On the other hand, M2, or alternatively activated macrophages, are induced by IL- 4 or IL-
13. M2-type macrophages have tumor growth-promoting properties and produce higher levels of immune-suppressive cytokines such as transforming growth factor-beta [34-36]. In addition, M2 macrophages are known to promote cancer cell invasion. Lewis et al. have reported that the macrophages migrating toward cancer cells are similar to M2 macrophages, and M2 macrophages promote prostate cancer progression and metastasis $[37,38]$.

The following line of argument suggests that the macrophages that were seen to migrate in the present study were M2 macrophages. Adipocytes can produce Th1 and Th2 cytokines, depending on the environment, and the balance of these cytokines determines macrophage polarization [39]. M2 polarization of macrophages around adipocytes can be provoked by Th2 cytokines such as IL-4 and IL-13 [40]. Hao et al. [41] have reported that M2 macrophages were produced by culturing RAW264.7 cells in a medium containing IL-4. IL-13 is produced in the adipose tissue of obese person and in the adipocytes of mice fed a high-fat diet. Moreover, macrophages were polarized into M2 macrophages by IL-13 contained in the conditioned medium of adipocytes [39]. In the present study, when RAW264.7 macrophages were treated with ATCM, which contains IL-4 and IL-13, expression of M2 macrophage markers such as arginase1, CD206, CXCL1, and IL-10 increased, and that of M1 markers such as TNF- $\alpha$, CXCL10, iNOS, IL-1 $\beta$, and IL-6 declined, thus showing that ATCM polarized M0 macrophages to $\mathrm{M} 2$ macrophages.

We also analyzed IL-4Ra expression in macrophages in response to ATCM. IL-4Ra is essential for signal transduction upon binding of either IL-4 or IL-13 [18]. We found that IL$4 \mathrm{Ra}$ protein significantly increased in macrophages treated with ATCM compared with those treated with ACM or TCM. Since the M2 macrophages seen in obesity are characterized by enhanced IL-4Ra expression [18], the increased IL-4Ra expression seen in this study suggests that the macrophages induced by ATCM were of M2 phenotype. Furthermore, IL-6 is linked to obesity [42] and primes macrophages for IL-4-dependent M2 polarization by inducing IL-4Ra expression in inflammatory conditions such as obesity [42]. In our study, when the adipocytes were treated with TCM, IL-6 production increased, suggesting that IL-6 promotes expression of IL-4Ra, but confirmation of this is needed.

Prostate cancers have a poor prognosis when they contain a large number of CD163-positive M2 macrophages [43,44]. M2 macrophages also promote the migration and invasion of 
cancer cells including PC3 prostate cancer cells by secreting growth factors and cytokines [45]. In the present study, MATCM increased the proliferation and invasiveness of prostate cancer cells, indicating that M2 macrophages can aggravate prostate cancers, which is consistent with the above reports [43-45].

In conclusion, the current study shows that the inflammatory reactions of prostate cancer cells treated with $T$. vaginalis increase the migratory ability and activation of adipocytes, and that factors secreted by the activated adipocytes stimulate macrophage migration and $\mathrm{M} 2$ polarization, and result in proliferation, and invasion of the prostate cancer cells. We propose that $T$. vaginalis infection contributes to the aggressiveness of prostate cancer in obese conditions by promoting the $\mathrm{M} 2$ polarization of macrophages.

\section{ACKNOWLEDGMENT}

This research was supported by the Basic Science Program through the National Research Foundation of Korea (NRF) funded by the Ministry of Science and ICT (NRF-2017R1A2B 4002072).

\section{CONFLICT OF INTEREST}

The authors declare no conflict of interest related to this study.

\section{REFERENCES}

1. Newman L, Rowley J, Vander Hoorn S, Wijesooriya NS, Unemo M, Low N, Stevens G, Gottlieb S, Kiarie J, Temmerman M. Global Estimates of the Prevalence and Incidence of Four Curable Sexually Transmitted Infections in 2012 Based on Systematic Review and Global Reporting. PLoS One 2015; 10: e0143304.

2. Gardner WA Jr. Culberson DE, Bennett BD. Trichomonas vaginalis in the prostate gland. Arch Pathol Lab Med 1986; 110: 430-432.

3. Mitteregger D, Aberle SW, Makristathis A, Walochnik J, Brozek W, Marberger M, Kramer G. High detection rate of Trichomonas vaginalis in benign hyperplastic prostatic tissue. Med Microbiol Immunol 2012; 201: 113-116.

4. Seo MY, Im SJ, Gu NY, Kim JH, Chung YH, Ahn MH, Ryu JS. Inflammatory response of prostate epithelial cells to stimulation by Trichomonas vaginalis. Prostate 2014; 74: 441-449.

5. Im SJ, Han IH, Kim JH, Gu NY, Seo MY, Chung YH, Ryu JS. Inflammatory response of a prostate stromal cell line induced by Trichomonas vaginalis. Parasite Immunol 2016; 38: 218-227.

6. Han IH, Kim JH, Kim SS, Ahn MH, Ryu JS. Signalling pathways associated with IL-6 production and epithelial-mesenchymal transition induction in prostate epithelial cells stimulated with Trichomonas vaginalis. Parasite Immunol 2016; 38: 678-687.

7. Siegel C. Re:Prostate cancer:prediction of biochemical failure after external-beam radiation therapy--Kattan nomogram and endorectal MR imaging estimation of tumor volume. J Urol 2012; 188: 432-433

8. Karan D, Thrasher JB, Lubaroff D. Prostate cancer: genes, environment, immunity and the use of immunotherapy. Prostate Cancer Prostatic Dis 2008; 11: 230-236.

9. Wolin KY, Carson K, Colditz GA. Obesity and cancer. Oncologist 2010; 15: 556-565.

10. Iyengar NM, Gucalp A, Dannenberg AJ, Hudis CA. Obesity and Cancer Mechanisms: Tumor Microenvironment and Inflammation. J Clin Oncol 2016; 34: 4270-4276.

11. Laurent V, Guérard A, Mazerolles C, Le Gonidec S, Toulet A, Nieto L, Zaidi F, Majed B, Garandeau D, Socrier Y, Golzio M, Cadoudal T, Chaoui K, Dray C, Monsarrat B, Schiltz O, Wang YY, Couderc B, Valet P, Malavaud B, Muller C. Periprostatic adipocytes act as a driving force for prostate cancer progression in obesity. Nat Commun 2016; 7: 10230.

12. Hefetz-Sela S, Scherer PE. Adipocytes:impact on tumor growth and potential sites for therapeutic intervention. Pharmacol Ther 2013; 138: 197-210.

13. Arendt LM, McCready J, Keller PJ, Baker DD, Naber SP, Seewaldt $\mathrm{V}$, Kuperwasser C. Obesity promotes breast cancer by CCL2-mediated macrophage recruitment and angiogenesis. Cancer Res 2013; 73: 6080-6093.

14. Sfanos KS, De Marzo AM. Prostate cancer and inflammation: the evidence. Histopathology 2012; 60: 199-215.

15. Lu Y, Cai Z, Xiao G, Liu Y, Keller ET, Yao Z, Zhang J. CCR2 expression correlates with prostate cancer progression. J Cell Biochem 2007; 101: 676-685.

16. Sica A, Schioppa T, Mantovani A, Allavena P. Tumour-associated macrophages are a distinct M2 polarised population promoting tumour progression:potential targets of anti-cancer therapy. Eur J Cancer 2006; 42: 717-727.

17. Wu HM, Zhu SL, He LJ, Liu YH, Xie D. Clinical significance of macrophage migration inhibitory factor in invasion of ovarian cancer. Ai Zheng 2009; 28: 1054-1060 (in Chinese).

18. Braune J, Weyer U, Hobusch C, Mauer J, Bruning JC, Bechmann I, Gericke M. IL-6 regulates M2 polarization and local proliferation of adipose tissue macrophages in obesity. J Immunol 2017; 198: 2927-2934.

19. Bortell R, Owen TA, Ignotz R, Stein GS, Stein JL. TGF beta 1 prevents the down-regulation of type I procollagen, fibronectin, and TGF beta 1 gene expression associated with 3T3-L1 pre-adipocyte differentiation. J Cell Biochem 1994; 54: 256-263.

20. Jang KS, Han IH, Lee SJ, Yoo J, Kim YS, Sim S, Ryu JS. Experimental rat prostatitis caused by Trichomonas vaginalis infection. Prostate 2019; 79: 378-389.

21. Kim JH, Kim SS, Han IH, Sim S, Ahn MH, Ryu JS. Proliferation of prostate stromal cell induced by benign prostatic hyperplasia 
epithelial cell stimulated with Trichomonas vaginalis via crosstalk with mast cell. Prostate 2016; 76: 1431-1444.

22. Kim JH, Han IH, Kim YS, Noh CS, Ryu JS. Proliferation of prostate epithelia induced by IL-6 from stroma reacted with Trichomonas vaginalis. Parasite Immunol 2018; 40: e12531.

23. Tokuda Y, Satoh Y, Fujiyama C, Toda S, Sugihara H, Masaki Z. Prostate cancer cell growth is modulated by adipocyte-cancer cell interaction. BJU Int 2003; 91: 716-720.

24. Finley DS, Calvert VS, Inokuchi J, Lau A, Narula N, Petricoin EF, Zaldivar F, Santos R, Tyson DR, Ornstein DK. Periprostatic adipose tissue as a modulator of prostate cancer aggressiveness. J Urol 2009; 182: 1621-1627.

25. Xie H, Li L, Zhu G, Dang Q, Ma Z, He D, Chang L, Song W, Chang HC, Krolewski JJ, Nastiuk KL, Yeh S, Chang C. Infiltrated pre-adipocytes increase prostate cancer metastasis via modulation of the miR-301a/androgen receptor (AR)/TGF-beta1/Smad/ MMP9 signals. Oncotarget. 2015; 6: 12326-12339.

26. Iyengar NM, Hudis CA, Dannenberg AJ. Obesity and cancer: local and systemic mechanisms. Annu Rev Med 2015; 66: 297-309.

27. Nonomura N, Takayama H, Nakayama M, Nakai Y, Kawashima A, Mukai M, Nagahara A, Aozasa K, Tsujimura A. Infiltration of tumour-associated macrophages in prostate biopsy specimens is predictive of disease progression after hormonal therapy for prostate cancer. BJU Int 2011; 107: 1918-1922.

28. Xu H, Barnes GT, Yang Q, Tan G, Yang D, Chou CJ, Sole J, Nichols A, Ross JS, Tartaglia LA, Chen H. Chronic inflammation in fat plays a crucial role in the development of obesity-related insulin resistance. J Clin Invest 2003; 112: 1821-1830.

29. Oh DY, Morinaga H, Talukdar S, Bae EJ, Olefsky JM. Increased macrophage migration into adipose tissue in obese mice. Diabetes 2012; 61: 346-354.

30. Ito $Y$, Ishiguro $H$, Kobayashi $N$, Hasumi $H$, Watanabe $M$, Yao M, Uemura H. Adipocyte-derived monocyte chemotactic protein-1 (MCP-1) promotes prostate cancer progression through the induction of MMP-2 activity. Prostate 2015; 75: 1009-1019.

31. Takahashi K, Mizuarai S, Araki H, Mashiko S, Ishihara A, Kanatani A, Itadani H, Kotani H. Adiposity elevates plasma MCP-1 levels leading to the increased CD11b-positive monocytes in mice. J Biol Chem 2003; 278: 46654-46660.

32. Galván GC, Johnson CB, Price RS, Liss MA, Jolly CA, deGraffenried LA. Effects of Obesity on the Regulation of Macrophage Population in the Prostate Tumor Microenvironment. Nutr Cancer 2017; 69: 996-1002.

33. Ruffell B, Affara NI, Coussens LM. Differential macrophage programming in the tumor microenvironment. Trends Immunol
2012; 33: 119-126.

34. Haase J, Weyer U, Immig K, Klöting N, Bluher M, Eilers J, Bechmann I, Gericke M. Local proliferation of macrophages in adipose tissue during obesity-induced inflammation. Diabetologia $2014 ; 57$ : 562-571.

35. Gordon S. Alternative activation of macrophages. Nat Rev Immunol 2003; 3: 23-35.

36. Kim D, Yoon JH, Kim J, Ryu SH. Adipokines involved in macrophage recruitment. In Leazek Szablewski ed, Glucose Homeostasis. London UK. IntechOpen. 2014, pp133-153.

37. Lewis CE, Pollard JW. Distinct role of macrophages in different tumor microenvironments. Cancer Res 2006; 66: 605-612.

38. Sica A, Larghi P, Mancino A, Rubino L, Porta C, Totaro MG, Rimoldi M, Biswas SK, Allavena P, Mantovani A. Macrophage polarization in tumour progression. Semin Cancer Biol 2008; 18: 349-355.

39. Kang K, Reilly SM, Karabacak V, Gangl MR, Fitzgerald K, Hatano $\mathrm{B}$, Lee CH. Adipocyte-derived Th2 cytokines and myeloid PPARdelta regulate macrophage polarization and insulin sensitivity. Cell Metab 2008; 7: 485-495.

40. Martinez FO, Gordon S. The M1 and M2 paradigm of macrophage activation:time for reassessment. F1000Prime Rep 2014; 6: 13.

41. Kwon H, Laurent S, Tang Y, Zong H, Vemulapalli P, Pessin JE. Adipocyte-specific IKKbeta signaling suppresses adipose tissue inflammation through an IL-13-dependent paracrine feedback pathway. Cell Rep 2014; 9: 1574-1583.

42. Mauer J, Chaurasia B, Goldau J, Vogt MC, Ruud J, Nguyen KD, Theurich S, Hausen AC, Schmitz J, Brönneke HS, Estevez E, Allen TL, Mesaros A, Partridge L, Febbraio MA, Chawla A, Wunderlich FT, Brüning JC. Signaling by IL-6 promotes alternative activation of macrophages to limit endotoxemia and obesity-associated resistance to insulin. Nat Immunol 2014; 15: 423-430.

43. Lanciotti M, Masieri L, Raspollini MR, Minervini A, Mari A, Comito G, Giannoni E, Carini M, Chiarugi P, Serni S. The role of M1 and M2 macrophages in prostate cancer in relation to extracapsular tumor extension and biochemical recurrence after radical prostatectomy. Biomed Res Int 2014; 2014: 486798.

44. Erlandsson A, Carlsson J, Lundholm M, Fält A, Andersson SO, Andrén O, Davidsson S. M2 macrophages and regulatory T cells in lethal prostate cancer. Prostate 2019; 79: 363-369.

45. Allavena P, Sica A, Solinas G, Porta C, Mantovani A. The inflammatory micro-environment in tumor progression: the role of tumor-associated macrophages. Crit Rev Oncol Hematol 2008; 66: 1-9. 
